\title{
Defining the Coronary Slow Flow Phenomenon
}

\author{
John F. Beltrame, MD, PhD
}

$\mathbf{T}$ he enigma of angina in the absence of significant epicardial coronary artery disease (CAD) has puzzled cardiologists since the advent of invasive coronary angiography. Indeed, an early paper by Likoff et $\mathrm{al}^{1}$ was entitled 'Paradox of normal selective coronary angiograms in patients considered to have unmistakable coronary heart disease' and described 15 women with angina, a positive exercise stress test and normal coronary angiography. This condition was later described as 'syndrome $\mathrm{X}$ ' following the demonstration of myocardial lactate production during rapid atrial pacing in these patients. Investigators attributed this effort-related myocardial ischemia (and thus angina) to coronary microvascular dysfunction, although controversy still exists as to the exact mechanisms responsible for the chest pain., ${ }^{2,3}$

\section{Article p 936}

The challenge for clinicians and researchers investigating patients with chest pain and insignificant coronary disease on angiography is to identify when the chest pain is cardiac in origin. The approach used to define and diagnose syndrome $\mathrm{X}$ is a positive exercise stress test. However, this would not necessarily identify all patients with coronary microvascular dysfunction, since most clinical cardiologists have endured the frustration of finding a patient with severe CAD despite a normal stress test.

An alternative approach to identifying patients with coronary microvascular dysfunction is to evaluate coronary angiographic blood flow during diagnostic angiography. This technique has been developed by the Thrombolysis In Myocardial Infarction (TIMI) group and extensively used in the context of acute myocardial infarction. The underlying premise for this methodology is that angiographic contrast flow mimics coronary blood flow and thus may be restricted in the presence of increased coronary resistance (either from a residual infarctrelated stenosis or the distal microvasculature). Thus in the setting of non-obstructed coronary arteries, delayed contrast opacification is considered a marker of increased microvascular resistance and is the hallmark of the coronary slow flow phenomenon (CSFP).

The CSFP remains a 'phenomenon' because it is defined on the basis of the angiographic findings of delayed distal vessel opacification in the absence of obstructive CAD. To be considered a clinical syndrome, the clinical attributes of this angiographic finding need to be elucidated. In the current issue of the Journal, Hawkins et al have published an important evaluation of the clinical features of patients with the CSFP. ${ }^{4}$
These important findings warrant further discussion in relation to the angiographic definition and the associated clinical features.

\section{Angiographic Definition of the CSFP}

Although in principle defining the CSFP angiographically is straightforward (ie, delayed distal vessel opacification), the operational definition used by various investigators has varied with differences in (1) the presence of normal or nearnormal angiography, (2) the definition of 'slow' angiographic flow, and (3) how many of the epicardial vessels exhibit 'slow flow'.

In syndrome $\mathrm{X}$, only patients with angiographically smooth normal coronary arteries are considered for this diagnosis, in the belief that they do not have atherosclerotic disease. With the advent of intravascular ultrasound it has been appreciated that they may have extensive coronary atherosclerotic disease despite a normal angiogram and indeed this has been demonstrated in the CSFP. ${ }^{5}$ Irrespective of the presence of atherosclerotic disease, the fundamental concept in coronary microvascular disorders is explaining the presence of angina/ischemia in the absence of obstructive lesions in the epicardial coronary arteries. Thus, some researchers have considered patients with non-obstructive minor lesions (ie, $<40 \%$ stenosis) in the diagnosis of the CSFP, ${ }^{6}$ while others have only considered those with angiographically smooth vessels. ${ }^{4}$ With both approaches, the delayed distal vessel opacification cannot be explained by obstructive epicardial disease.

Two approaches have been used by investigators to define the threshold contrast flow that constitutes 'slow flow'. The TIMI flow grade is a semiquantitative index that scores epicardial artery contrast flow from TIMI-0 (no flow) to TIMI-3 (normal flow). TIMI-2 flow involves delayed filling of the distal vessels and is considered present when 3 or more beats are required to opacify the distal vasculature. This approach is frequently used in diagnosing the no-reflow phenomenon and has also been used to diagnose the CSFP. ${ }^{6}$ An alternative approach is to use the TIMI frame count method as described by Gibson et al. ${ }^{7}$ This quantitative method assesses the number of cine frames required to opacify the distal vasculature, with a reference value of $21 \pm 3$ frames established by these investigators. ${ }^{7}$ Thus, some researchers have defined the CSFP as any frame account above the baseline threshold, ${ }^{5}$ while others have used a threshold of more than 2 standard deviations above this reference value. ${ }^{4}$ It is noteworthy that studies using TIMI-2 flow as the diagnostic criterion for the CSFP typically report mean TIMI frame counts $>50$ frames. ${ }^{6}$ There

The opinions expressed in this article are not necessarily those of the editors or of the Japanese Circulation Society.

Received February 13, 2012; accepted February 13, 2012; released online February 25, 2012

Discipline of Medicine, The Queen Elizabeth Hospital, The University of Adelaide, Adelaide, South Australia, Australia

Mailing address: John F. Beltrame, Professor, BSc, BMBS, FRACP, PhD, Discipline of Medicine, The Queen Elizabeth Hospital, The

University of Adelaide, 28 Woodville Rd, Woodville South, South Australia 5011, Australia. E-mail: john.beltrame@adelaide.edu.au

ISSN-1346-9843 doi:10.1253/circj.CJ-12-0205

All rights are reserved to the Japanese Circulation Society. For permissions, please e-mail: cj@j-circ.or.jp 
Table. Defining a New Primary Coronary Microvascular Disorder: Proposed Criteria for Primary Coronary Slow Flow

\section{Angiographic evidence of the CSFP, defined by:}

a. No evidence of obstructive epicardial CAD (ie, no angiographic lesions $\geq 40 \%$ )

b. Delayed distal vessel contrast opacification as evidenced by either:

(i) TIMI-2 flow (ie, requiring $\geq 3$ beats to opacify the vessel), or

(ii) corrected TIMI frame count $>27$ frames (images acquired @ 30 frames/s)

c. The delayed distal vessel opacification is in at least 1 epicardial vessel

2. Exclusion of secondary causes of the CSFP, including:

a. No-reflow phenomenon

b. Coronary emboli

c. Coronary ectasia

d. Exogenous vasoconstrictor administration (eg, cocaine)

CSFP, coronary slow flow phenomenon; CAD, coronary artery disease; TIMI, Thrombolysis In Myocardial Infarction

are merits and limitations to both of these approaches, but both objectively identify a population with delayed vessel opacification.

There is also variation between studies in the number of vessels required to exhibit the delayed opacification for the diagnosis of the CSFP. Some studies consider the diagnosis when only 1 vessel fulfils the criterion, ${ }^{4,6}$ while others require all 3 vessels to be involved. Independent of these 2 approaches, delayed opacification in all 3 coronary arteries has been reported in affected patients, even though some vessels may not absolutely fulfill the CSFP threshold. ${ }^{8}$

An additional criterion used by Hawkins et al to define their CSFP cohort was to exclude patients with an impaired left ventricular ejection fraction. ${ }^{4}$ The rationale for this approach is to exclude myocardial conditions that may be associated with coronary microvascular dysfunction, ${ }^{9}$ in order to specifically identify patients with a primary coronary microvascular disorder (ie, not associated with myocardial or CAD). Nevertheless, this approach would exclude patients with a primary coronary microvascular disorder that resulted in a myocardial infarct, thereby potentiating a selection bias in the study population. However, other more inclusive studies have reported an abnormal left ventricular systolic function in only $6 \%$ of the CSFP patients. ${ }^{6}$

In order to define a population of patients with primary microvascular dysfunction, other causes of the angiographic phenomenon must be considered. For example, inadvertent injection of microbubbles during the angiographic procedure will impede the microcirculation, producing a transient CSFP. Similarly, the no-reflow phenomenon arises from distal embolization of an atherosclerotic lesion, as well as the release of vasoactive substances, resulting in the angiographic appearance of the CSFP. Large ectatic coronary arteries may give rise to the appearance of the CSFP because of a capacitance effect of filling the large vessels. When defining a primary coronary microvascular condition, these secondary causes must be excluded. Hence the term 'primary CSFP' and 'secondary CSFP' have been coined. ${ }^{6}$ With the focus on primary CSFP as a primary coronary microvascular disorder, the clinical features associated with this angiographic phenomenon are addressed below.

\section{Clinical Features of the CSFP}

The prevalence of the CSFP has been reported as $5.5 \%$ of diagnostic coronary angiograms in the current study, ${ }^{4}$ where the phenomenon is defined as normal angiography with a TIMI frame count $>27$ in at least 1 vessel. Although previous stud- ies $^{6}$ have reported only a $1 \%$ prevalence, a more recent systematic evaluation by this group suggests that it is approximately $3 \% .{ }^{10}$

Several studies have compared the clinical features of patients with normal angiography and the CSFP with a control group without the phenomenon. Despite differences in the angiographic definitions, several clinical attributes recur in these studies. Firstly, unlike syndrome X where there is a predilection among women, the CSFP is more common in $\mathrm{men}^{4,6}$ or equally distributed between the sexes. ${ }^{11,12}$ Secondly, the CSFP patients tend to be obese, ${ }^{4,12}$ with some reporting a predisposition to the metabolic syndrome. ${ }^{12}$ Another distinguishing characteristic of the CSFP is its association with rest or mixedpattern angina rather than exertional angina, as occurs in syndrome $\mathrm{X}$. As a consequence, many patients with CSFP undergo their index diagnostic angiography following an acute coronary syndrome presentation and this led to the concept of stable and unstable primary microvascular angina, such as syndrome $\mathrm{X}$ and the CSFP, respectively. ${ }^{13}$

Considering the clinical differences between syndrome $\mathrm{X}$ and the CSFP, a number of investigators have suggested that the CSFP should be considered a specific disease entity, ${ }^{6,14,15}$ with some suggesting it should be referred to as 'syndrome $\mathrm{Y}^{\prime} .{ }^{14} \mathrm{With}$ the study published in this issue of the Journal, ${ }^{4}$ the evidence base for the CSFP is expanding so that it may be soon considered a clinical syndrome rather than a phenomenon. However, if this field of research is to advance, a consensus statement to formalize the angiographic definition of the CSFP is required. As a prelude to this important development, the accompanying Table proposes a working definition for this intriguing disorder, which should be discussed at future scholarly forums.

\section{References}

1. Likoff W, Segal BL, Kasparian H. Paradox of normal selective coronary arteriograms in patients considered to have unmistakable coronary heart disease. N Engl J Med 1967; 276: 1063-1066.

2. Beltrame JF. Advances in understanding the mechanisms of angina pectoris in cardiac syndrome X. Eur Heart J 2005; 26: 946-948.

3. Maseri A, Beltrame JF, Shimokawa H. Role of coronary vasoconstriction in ischemic heart disease and search for novel therapeutic targets. Circ J 2009; 73: 394-403.

4. Hawkins BM, Stavrakis S, Rousan TA, Abu-Fadel M, Schechter E. Coronary slow flow: Prevalence and clinical correlations. Circ J 2012; 76: $936-942$.

5. Cin VG, Pekdemir H, Camsar A, Ciçek D, Akkus MN, Parmaksýz $\mathrm{T}$, et al. Diffuse intimal thickening of coronary arteries in slow coronary flow. Jpn Heart J 2003; 44: 907-919.

6. Beltrame JF, Limaye SB, Horowitz JD. The coronary slow flow 
phenomenon: A new coronary microvascular disorder. Cardiology 2002; 97: 197-202.

7. Gibson CM, Cannon CP, Daley WL, Dodge JT Jr, Alexander B Jr, Marble SJ, et al. TIMI frame count: A quantitative method of assessing coronary artery flow. Circulation 1996; 93: 879-888.

8. Beltrame JF, Limaye SB, Wuttke R, Horowitz JD. Coronary hemodynamic and metabolic studies of the coronary slow flow phenomenon. Am Heart J 2003; 146: 84-90.

9. Beltrame JF, Crea F, Camici P. Advances in coronary microvascular dysfunction. Heart Lung Circ 2009; 18: 19-27.

10. Jesuthasan LSB, Beltrame JF, Marwick TH. Incidence of coronary slow flow in a large teaching hospital. Heart Lung Circ 2009; 18: S121.

11. Xia S, Deng SB, Wang Y, Xiao J, Du JL, Zhang Y, et al. Clinical analysis of the risk factors of slow coronary flow. Heart Vessels 2011; 26: 480-486.

12. Yilmaz H, Demir I, Uyar Z. Clinical and coronary angiographic characteristics of patients with coronary slow flow. Acta Cardiol 2008; 63: 579-584.

13. Lanza GA, Crea F. Primary coronary microvascular dysfunction: Clinical presentation, pathophysiology, and management. Circulation 2010; 121: 2317-2325.

14. Leone MC, Gori T, Fineschi M. The coronary slow flow phenomenon: A new cardiac "Y" syndrome? Clin Hemorheol Microcirc 2008; 39: $185-190$.

15. Li JJ, Wu YJ, Qin XW. Should slow coronary flow be considered as a coronary syndrome? Med Hypotheses 2006; 66: 953-956. 